\title{
Application of allograft bone transplantation in bone tumour surgery
}

\author{
Rui Jiang ${ }^{1}$, Ziyan Zhang ${ }^{2}$, Han $\mathrm{Wu}^{1}$ and Chao Zhang ${ }^{3}$ \\ ${ }^{1}$ Department of orthopedics, China-Japan Union Hospital of Jilin University, Jilin 130033, China \\ ${ }^{2}$ Department of orthopedics, The Second Hospital of Jilin University, Jilin 130041, China \\ ${ }^{3}$ Department of ophthalmology, The Second Hospital of Jilin University, Jilin 130041, China \\ Corresponding Author: Chao Zhang: zhangchao0701@163.com
}

Keywords: allograft bone; transplantation; rejection reaction; bone tumour

\begin{abstract}
Objective: To discuss the scope, effect, rejection reaction, etc. of applying allograft bone transplantation in bone tumour surgery. Method: 120 cases of allogeneic bone transplantation were used in bone tumour surgery from October 1983 to March 2003, including 102 cases of tumor curettage and cancellous allogenic bone transplantation; 12 cases of tumor curettage, allograft massive cortical bone and cancellous bone transplantation; 2 cases of bone tumor segmental resection and allograft femoral shaft transplantation; 3 cases of bone tumor segmental resection and hemiarthrosis transplantation and 1 case of tumor excision and massive allograft sacrum transplantation. Results: 2 of 120 cases had minor rejection reaction only, who were mostly manifested as post-operative incision exudation, exudation was stopped and the incision was healed three months later, incision, and other cases are healed by the first intention. Conclusion: allograft bone has wide application scope, excellent effect and rare rejection reaction in bone tumour surgery. It is important in bone defect fillers.

Currently, bone defect fillers are more and more. Common fillers include autogenous bone, allogeneic bone, artificial bone, artificial prosthesis, bone cement, etc. What is the position of allogeneic bone among various bone defect fillers?

How about application scope, effect, rejection reaction, etc. in bone tumour surgery especially? In the paper, 120 cases of allogeneic bone transplantation in our department from October 1983 to March 2003 are summarized. The above issues are discussed.
\end{abstract}

\section{Data and method}

\subsection{General data}

120 cases in the group include 75 male cases and 45 female cases. They are 8 to 62 old years with average age of 26 years old. Tumor types: 25 cases of giant-cell tumor; 18 cases of solitary bone cyst; 13 cases of non-ossifying fibroma; 10 cases of enchondroma; 9 cases of aneurysmal bone cyst; 7 cases of fibrous dysplasia; 6 cases of chondromyxoid fibroma; 5 cases of fibrous dysplasia; 5 cases of Juxta-articular bone cyst; 5 cases of osteoidosteoma; 3 cases of benign chondroblastoma; 3 cases of eosinophilic granuloma; 3 cases of osteosarcoma and 1 case of metastatic tumor. Tumor site: 48 cases in femur; 33 cases in tibia; 15 cases in humerus; 5 cases in ilium; 4 cases in ulna; 3 cases in fibula; respectively 2 cases in phalanx and astragalus as well as 1 case in sacrum.

\subsection{Operation method}

Tumor curettage and cancellous allogenic bone transplantation were implemented for 102 cases; tumor curettage, allograft massive cortical bone and cancellous bone transplantation were implemented for 12 cases; bone tumor segmental resection and allograft femoral shaft transplantation were implemented for 2 cases; bone tumor segmental resection and hemiarthrosis transplantation were implemented for 3 cases; tumor excision and massive allograft sacrum transplantation were implemented for 1 case.

\subsection{Allogeneic bone preservation method}

95\% ethyl alcohol was used for preserving cancellous bone, and microfuge was used for 
preserving allograft femoral shaft and hemiarthrosis before 2001. FDBA prepared by bone bank of our department was used after 2001.

1.4 Typical cases

1.4.1 1 male case was 12 years old, and he suffered from right femur middle-section ossifying fibroma complicated with pathological fracture. He accepted bone tumor segmental resection, allograft femoral shaft transplantation and steel plate internal screw fixation on December 6, 1990. Porosis was discovered 3 months after operation, fracture line became fuzzy 3 months after operation, and fracture line disappeared and marrow cavity was penetrated 1 year after operation.

1.4.2 2 female cases were 16 years old, they suffered from left tibia proximal osteosarcoma, and the cases accepted bone tumor segmental resection, hemiarthrosis transplantation and gastrocnemius medial heal metastasis on September 29, 1993. Incision was healed by the first intention 2 weeks after operation. Fracture line disappeared, and marrow cavity was penetrated 2 years after operation. The hemiarthrosis $\mathrm{X}$ ray 4 years and 7 years after operation was basically the same as that 2 years after operation. The cases did not have local recurrence 10 years after operation, and knee joint can be bent for $90^{\circ}$.

\section{Results}

2 of 120 cases with allogeneic bone transplantation had minor rejection reaction. The following clinical manifestations were discovered: incision was exuded 2 weeks after operation, exudation was stopped, and incision was healed 2 months later. Other 118 cases did not have rejection reaction in any form, and the incisions were healed by the first intention.

\section{Discussion}

\subsection{Position of allogeneic bone in bone defect fillers}

Currently, bone defect fillers are more and more. Common fillers include autogenous bone, allogeneic bone, artificial bone, artificial prosthesis, bone cement, etc. The author adopts autologous or cancellous allogenic bone and imported Austine replacement bone aiming at small bone defect; massive allogeneic bone is adopted aiming at big bone defect [1]; hemiarthrosis is adopted for cases with young age aiming at malignant tumor around knee joint, older cases undergo artificial prosthesis replacement [2]. Therefore, it can be concluded that allogeneic bone at least still occupy an important position in bone defect filler till present.

\subsection{Prognosis after allogeneic bone transplantation}

In one orthopaedic seminar, some people proposed the question about the survival time after allogeneic bone implantation. Obvious, the proposal is not exact since Phemiter calls the histology change process after allogeneic bone implantation as 'creeping substitution' as early as 1914 . The statement is still adopted till present. The basic process of bone healing is creeping substitution for both allogeneic bone transplantation and autogenous bone transplantation without blood supply. Bone surface extension is utilized for osteogenesis during creeping substitution and bone conduction. Therefore, it is easy to regard creeping substitution as bone conduction. Bone conduction is regarded as the only approach of fracture healing. In fact, creeping substitution and bone conduction are not synonyms. Creeping bone conduction also can be discovered on the implantation artificial prosthesis surface, but replacement is not accompanied. However, bone surface not only has bone conduction, but also suffers from bone induction caused by bone surface absorption and BMP release. Therefore, creeping substitution should be regarded as the joint result of bone conduction and bone induction exactly [3]. All time of replacing sequestrum by vitalized bone depends on patient age, bone graft site and bone graft category. Shorter time is required for children. The process can be completed within one year for some children. Relatively longer time is required for adults, and some patients even spend more than 10 years. The group contains one case, and marrow cavity was penetrated one year later.

3.3 Rejection after allogeneic bone implantation

Allograft bone transplantation is different from other solid organ transplantation processes. 
Allograft bone belongs to implant rather than transplant according to strict meaning. Immune rejection result shows that transplantation bone survival cells are killed, it is mainly manifested as follows: transplantation bone is absorbed without creeping substitution restoration and blood circulation reconstruction. The research of Friedaender shows that humoral immunity does not play an important role in allograft bone transplantation immune rejection reaction. Currently, it is generally believed that cell immunity plays a dominated mode in rejection reaction [4]. Horowitzh Friedlaender proposed a hypothesis that allograft bone transplantation immune rejection reaction can be divided into 2 stages, the $1^{\text {st }}$ stage is similar to rejection reaction of solid organ transplantation; the $2^{\text {nd }}$ stage is manifested as cytokine generation by activated immune cells, the cytokines activate osteoclasts, thereby leading to transplantation bone absorption [ 5]. In fact, the rejection reaction as serious as solid organ transplantationis not discovered clinically. 2 of 120 cases with allograft bone transplantation in the group have rejection reaction with manifestation of more post-operative incision exudation, exudation is stopped, and incision is healed 3 months later.

3.4 Characteristics of allograft bone: autogenous bone can not meet requirements as well as young and old patients with poor surgery tolerance aiming at major bone defect. It is suitable to adopt allogeneic bone transplantation especially for patients with massive bone defect and cartilago articularis defect, ilium transplantation and cancellous bone can not solve the problems in the aspects of load bearing and articular surface reconstruction only. Allograft bone sources are wide. Bone strength can be completely preserved. The shape and size can be matched with receptor bone [10], immunogenicity is low, ligament attachment also can be reserved for soft tissue reconstruction. In addition, allogeneic bone joint also has applicable joint surface for joint reconstruction [11-13]. Allogeneic bone has bone induction and bone conduction, which can reach the requirements of bone healing, and it is consistent with the requirements of biomechanics through moulding [12]. It can be used for the whole life if no complication is available. It also can be combined with artificial prosthesis for reconstructing the limbs more perfectly. In the experiment, massive allogeneic bone can be adopted for restoring bone defect, it is proved through follow-up observation that rejection reaction is mild after massive allogeneic bone transplantation, it can reach solid healing and has no different from normal bone structure after certain time, and the joint function is excellent.

\section{Healing process and characteristics in large-section allograft bone transplant}

Allogeneic bone sources are relatively rich, China bone bank is rapidly development especially in recent years, and allogeneic bone sources are more convenient as a result. Allogeneic bones preserved by different methods have different immunogenicity and bone induction ability. The histology change belongs to a creeping substitution process after autogenous bone transplantation, transplantation bone mainly plays the role of bone induction and bone conduction. Many scholars proves that the changes are the same after allogeneic bone transplantation through experiment. However, bone induction ability is lower than that of autogenous bone. Cancellous bone transplantation, regardless of autologous or allograft bones, is combined with host bone through formation of new bones on transplantation bone trabecula. It is called 'sandwich' phemomena. Host's new bone is deposited on cancellous allogenic bone, as a skeleton. Most (not all) transplantation bones are finally absorbed, thereby host bones replace transplantation bone [7]. In clinical cases, when allogeneic bone is used for massive implantation or total (semi) joint transplantation, and the healing time is prominently later than autogenous bone implantation. However, the healing time after mere cancellous allogenic bone implantation is not reported. The healing time of patients in the group is 4.5 months, which is similar to autologous cancellous bone implantation. The reason is shown as follows: the revascularization of the former implantation is difficult. The later cancellous bone grid structure is more suitable for creeping substitution. Experimental study shows that allogeneic bone transplantation is similar basically to healing process of autogenous bone transplantation. However, the allogeneic bone healing speed is slow. The bone healing time of joint part is averagely 15 months aiming at $8 \%$ cases in the group. The healing time is longer. The healing of the joint part does not mean that the transplantation bone section has been activated. Bone absorption and creeping substitution still survive continuously in 
the body. The figure shows that the bone absorption of transplantation bone section is still clear and visible after healing of the joint part.

Allograft bone undergoes pathological examination. It is discovered that internal restoration of implantation bone is very slow, only $8 \%<$ of transplantation bones are replaced by new bones within 9 years after transplantation, and the deep parts without restoration still keep the original structure. Post-operative results of 8 patients in the group show that: allogeneic bone section biopsy is carried out annually, and the above viewpoint is also supported. We design condylar interlocking intramedullary nail according to the healing characteristics, bone section collapse can be effectively prevented during active bone absorption, thereby leading to bone section fracture or internal fixation failure (nail bending, nail breakage, etc.). The healing process of transplantation bone in the body does not merely depend on host to carry out creeping substitution to transplantation bone, but multi-path activated healing process in transplantation bone section. Namely, creeping substitution and bone induction are carried out at the same time aiming at all transplantation bone surfaces with contact to host bone end or transplantation bone bed soft tissue. According to allogeneic bone transplantation animal experiment, we prove that the activation process of allogeneic bone in the body is multi-path, namely activation process is realized from host bone end to transplantation bone, from transplantation bone surrounding area to transplantation bone center and from original bone canalicules and Haversian canal of transplantation bone to surrounding area. The healing mainly depends on bone conduction, and bone induction also plays an active role, (it should be further discussed in other paper). Li Yafei, etc. observe long-term prognosis after large-section allogeneic bone transplantation. It is discovered that bone induction plays a role in early new bone replacement and healing of bone transplantation, and there have been blood supply and osteogenesis. Therefore, it can be predicted that transplantation bone suffers from osteogenesis due to surrounding host invasion.

Femur distal end undergoes hemiarthrosis transplantation. Post-operative long-term follow-up observation shows that allogeneic bone healing mainly depends on massive mesenchymal cell invasion around allogeneic bone, which is accompanied with consistent neoplastic bone formation. In the surgery, we adopt bone stump eriosteal sleeve or myocutaneous flap coverage transplantation, steel plate fixation should not be used as far as possible but intramedullary nail fixation, which is beneficial for increasing the contact surface of transplantation bone and host. Sound conditions are created for allogeneic bone healing. Rejection reaction of allogeneic bone implantation and its secondary infection belong to main complications. Most scholars believe that its immunogenicity mainly comes from bone marrow cell and bone cell surface. Mveloid tissues are removed in the operation for reducing its immunogenicity [7]. The rejection reaction infection rate after massive allogeneic bone implantation or total (semi) joint transplantation is $10 \%$ to $30 \%$ [ 8]. Only two cases in the group suffer from infection. The cases belong to calcaneus post-operative wound skin edge necrosis secondary infection, which should be related to rejection reaction. The cases in the group do not suffer from rejection reaction possibly because blood supply around bone defect cavity is sound during cancellous bone implantation and cell components can be removed more completely aiming at implanted cancellous bone through repeated flushing and immersion. Cell components can not be removed completely during Massive allogeneic bone implantation or total (semi) joint transplantation, general cancellous bone bone graft should be less than massive implantation or total (semi) joint transplantation.

\section{Influence of internal fixation on large-section allogeneic bone healing}

Some scholars discover that steel plate internal fixation is more prone to allogeneic bone fracture than intramedullary nail fixation. Firm intramedullary nail fixation can prominently reduce the complication in allogeneic bone healing process. In the group, bone ununion rate of ordinary intramedullary nail and interlocked intramedullary nail fixation is compared, and the later is prominently reduced. Therefore, we believe that firm intramedullary nail fixation has more biomechanics advantages in the process of transplantation bone healing, which is beneficial for allogeneic bone healing. In summary, deep frozen allograft bone is used for restoring bone defect 
bone tumour tumor resection. It is an actually feasible and effective method. Its healing process in the body is a multi-path activation process, which is affected by internal fixation and many other factors. Mere autogenous bone transplantation can not reach clinical requirements in the aspects of shape, size, bone graft, mechanical support and other aspects. Mere long-section allogeneic bone transplantation has poor bone induction function, slow bone healing speed and many complication. Anastomotic vessel autologous fibula is overlapped in long-section allograft bone medullary cavity, the compound bone transplantation can form a 'comprehensive and multi-mode' healing: transplanted autologous fibula and host bone healing, allogeneic bone and host bone combined end healing, allogeneic bone and autologous fibula healing in allogeneic bone medullary cavity; activation is realized from transplantation bone original bone canalicules and haversian canal to surrounding area, thereby realizing comprehensive and muti-mode revascularization and new bone formation. Xu Zhonghe, etc. [6] fibula transplantation with monitoring flap provides a reliable method for blood supply monitoring of transplantation post-operative fibula. It is simply and directly ensured that the transplanted fibula belongs to 'vital bone' through monitoring flap homologous with fibular blood vessels. Therefore, we believe that allogeneic bone is stored and treated correctly before operation, its contact area with host is increased in the operation through effective approach, thereby maintaining sound biomechanical characteristics, functions are exercised rationally after operation, and it is critical for improving surgery effect and reducing complications.

5.1 Allogeneic bone fracture; Allogeneic bone fracture is one of the most important complications in allograft bone transplantation, which mostly occur six months after allogeneic bone transplantation. It reaches the peak two two three years later. It is related to transplantation bone revascularization speed according to time analysis. Creeping substitution and bone block revascularization are implemented after allogeneic bone transplantation, and osteoclast absorption is better than new osteogenesis [9-10]. If support protection is not available after operation, early or too heavy load must lead to fracture due to transplantation bone stress concentration. Severe clinical symptoms can not be caused after allogeneic bone fracture. The function influence is low. The joint stability is reduced.

Allograft bone sources are wide, which have excellent structures and strengths as support materials, which can be shaped and implanted easily. It can be permanently healed with the host after successful transplantation. Allogeneic bone still has certain immunity after a series of treatments, thereby leading to rejection reaction and influence on revascularization and creeping substitution. There are some complications after operation. A lot of clinical practice and studies show that allograft bone transplantation is not worse than other alternatives, which can be regarded as an important reconstruction mode for bone tumour post-operative bone defects.

\section{Acknowledgments}

This work was supported by The study of c-Myc and mTOR inhibitors in the combined targeting therapy of osteosarcoma.

\section{References}

[1] Salai M,Dudkiewitz I,Amit Y,et al. Versatile Utilization of Massivem Bone Allografts in Orthopedic Surgery. Cell Tissue Bank.2000;1(3):223-227.

[2] Wuisman P,Gohlke F,Witlox A. Allografts in reconstruction of osseous defects in primary malignant bone tumors. Orthopade.2003;32(11):994-1002.

[3] Mankin HJ,Gebhardt MC,Jennings LC,et al. Long-term results of allograft replacement in the management of bone tumors. ClinOrthop Relat Res. 1996;(324):86-97.

[4] Muscolo DL,Ayerza MA,Aponte-Tinao LA,et al. Use of distal femoral osteoarticular allografts in limb salvage surgery. J Bone Joint Surg Am. 2005;87(11):2449-2455. 
[5] Matejovsky Z Jr,Matejovsky Z,Kofranek I. Massive allografts in tumour surgery. Int Orthop. 2006;30(6):478-483.

[6] Itiravivong P,Tejapongvorchai T,Kuptniratsaikul S. Allograft replacement in limb salvage surgery for bone tumors. J Med AssocThai. 2001;84 Suppl 1:S396-400.

[7] Liu JZ,Wang Z,Li MQ. Allografting for massive bone defect:bone and soft tissue reconstruction and postoperative rehabilitation. Zhongguo Xiu Fu Chong Jian Wai Ke Za Zhi. 2001;15(4):244-247.

[8] Muscolo DL,Ayerza MA,Aponte-Tinao LA. Massive allograft use in orthopedic oncology. Orthop Clin North Am. 2006;37(1):65-74.

[9] Huang TL,Chen TH,Chen WY,et al. Allograft arthrodesis of the knee in high-grade osteosarcoma. J Chin Med Assoc.2005;68(9):425-430.

[10] Delloye C,Banse X,Brichard B,et al. Pelvic reconstruction with a structural pelvic allograft after resection of a malignant bone tumor. J Bone Joint Surg Am. 2007;89(3):579-587.

[11] Langlais F,Lambotte JC,Thomazeau H. Long-term results of hemipelvis reconstruction with allografts. Clin Orthop Relat Res.2001;(388):178-186.

[12] Muscolo DL,Ayerza MA,Aponte-Tinao LA,et al. Unicondylar osteoarticular allografts of the knee. Surgical technique. J Bone Joint Surg Am. 2008;90 Suppl 2 Pt 2:206-217.

[13] Kharrazi FD,Busfield BT,Khorshad DS,et al. Osteoarticular and total elbow allograft reconstruction with severe bone loss. ClinOrthop Relat Res. 2008;466(1):205-209. 